\title{
Relación Entre La Sensibilidad Al Premio Y Al Castigo Y Los Niveles Motivacionales. Estudiantes Superiores De Música, Instrumentistas De Viento Metal
}

\author{
Francisco Javier Zarza-Alzugaray \\ Universidad Complutense de Madrid, Spain
}

Doi: 10.19044/esj.2018.v14n11p23 URL:http://dx.doi.org/10.19044/esj.2018.v14n11p23

\begin{abstract}
The sensitivity to reward and punishment, within the theory of anxiety of Gray (1986) is considered as one of the ways to explain different motivational aspects and activators or inhibitors of behavior. In this sense, in this article we present how from the two activation or inhibition of behavior ways we can see how the motivation of a sample of students of higher level wind instruments is modulated and explained. Thus, the specific demands of task related together with the sensitivity to punishment are the factors that have the greatest explanatory and associative power, while factors such as sensitivity to punishment or perfectionism need further study to clarify their role in the explanation of the general motivation and of the presence of behavior activation. Finally, a better knowledge of these constructs will allow the educational community to improve the teaching practice.
\end{abstract}

Keywords: Music education, music students, sensitivity to reward, sensitivity to punishment, achieve motivation

\section{Resumen}

La sensibilidad al premio y al castigo, dentro de la teoría de ansiedad de Gray (1986) es considerada como una de las vías para la explicación de distintos aspectos motivacionales y activadores o inhibidores de la conducta. En este sentido, en este artículo presentamos cómo a partir de las dos vías de activación o inhibición de la conducta podemos ver cómo se modulan y explica la motivación de una muestra de estudiantes de instrumentos de viento metal de nivel superior. Así, las demandas específicas de tarea relacionadas junto con la sensibilidad al castigo son los factores que mayor potencia explicativa y asociativa mientras que factores como la sensibilidad al castigo o el perfeccionismo necesitan de más estudio para aclarar su papel en la explicación de la motivación general y de la presencia de activación de 
conductas. El fin último es un mejor conocimiento de estos constructos ya que permitirán a la comunidad educativa la mejora de la práctica docente.

Palabras clave: educación musical, estudiantes de música, sensibilidad al premio, sensibilidad al castigo, motivación de logro

\section{Introducción}

Desde esta investigación se ha asumido como punto de partida para el estudio de la motivación hacia el logro el modelo de Weiner (1986a, 1986b, 2000), el cual postula que la motivación es iniciada a través de un resultado conductual determinado; éste es interpretado por la persona como éxito (consecución del logro) o fracaso (no consecución del logro), y se relaciona en una primera instancia con los sentimientos de felicidad o tristeza y frustración, respectivamente.

En el caso de los músicos con éxito profesional, de todos los tipos, están caracterizados por un deseo de mejora de sus actuaciones y sus habilidades. Es sabido que los músicos invierten mucho tiempo practicando, si bien es su profundo deseo de mejora lo que hace que ésta sea efectiva (Ericsson, Krampe y Tesch-Römer, 1993; Shehan-Campbell, 1995; Walser, 1993).

De este modo, se consideran indispensables para la comprensión del modelo, las dimensiones de lugar de causalidad, estabilidad, y controlabilidad; así como sus consecuencias relacionadas con las expectativas y las emociones que a la postre desencadenan las conductas futuras con unas determinadas características como intensidad, latencia, persistencia, dirección para, en fín último la consecución de un logro determinado y la consiguiente mejora o empeoramiento de la motivación ante retos futuros semejantes (Manassero y Vázquez, 1998).

Desde esta perspectiva, Weiner (1986a, 1986b, 2000) incluye en este modelo todas las conductas humanas y procesos cognitivos que influyen sobre la conducta de manera consciente, obteniendo así un modelo integrador y completo para la explicación del constructo de la motivación de logro. Se puede decir que en este modelo se buscan leyes y principios generales, capaces de explicar tanto los aspectos racionales como los aparentemente irracionales de la motivación (Manassero y Vázquez, 1998).

Con este modelo se puede asumir que el marco teórico de la conceptualización de la teoría de motivación de logro consiste, en definitiva, en lograr un dominio de la tarea, influenciado por la existencia de una disposición conductual, bien de aproximación, bien de evitación para la consecución del logro (Elliot y Church, 1997). Esta disposición conductual está a su vez influenciada por las diferentes explicaciones atributivas que se le puedan dar a dichos logros o fracasos por la consecución del objetivo. 
En este sentido vemos que son varias las teorías que contemplan la idea de la existencia de una motivación apetitiva o de comportamientos de aproximación liderados por lo que se ha denominado como sistema de activación del comportamiento (Cloninger, 1987; Fowles, 1980; Gray, 1981, 1987, 1990, 1994a, 1994b), sistema de adquisición de compromisos (Depue, Krauss y Spoont, 1987) o sistema de comportamientos facilitadores (Depue y Collins, 1999).

En el lado opuesto encontramos autores que atribuyen la existencia de motivaciones aversivas y comportamientos de evitación y/o escape a lo que normalmente se ha denominado como sistema de inhibición del comportamiento (Cloninger, 1987; Gray, 1981, 1987, 1990, 1994b) o sistema de retirada, (Davidson, 1984, 1988, 1995, 1998). Se cree que ambos sistemas tienen sustratos neurológicos diferenciados y ejercen distintas y marcadas influencias en las diferentes acciones (Carver, 2006).

El modelo de ansiedad de Gray (1986) presenta un enfoque de la ansiedad reduccionista a dos factores desde el prisma biológico. Del mismo modo que Eysenck (1985), postula que en la base de las diferencias individuales de personalidad, se encuentran los mecanismos biológicos sobre los que se sostienen los procesos de aprendizaje, emoción y motivación (Hernangómez y Fernández, 2012).

Gray, a partir de Eysenck, propone dos dimensiones básicas de la personalidad, ansiedad e impulsividad, las cuales surgen de la combinación de los factores de Eysenck (Hernangómez y Fernández, 2012; Pinto-Meza et al., 2009).

Partiendo de la base anteriormente descrita, Gray $(1981,1982)$ teoriza en su modelo de personalidad que existen diferentes estructuras o sistemas cerebrales, que influyen de manera sensible en el reforzamiento de conductas y en el control de las emociones (Torrubia, Ávila, Moltó y Caseras, 2001). Estos sistemas son denominados por la comunidad científica como el Sistema de Inhibición Conductual, (Behavioural Inhibition System, BIS en inglés), y el Sistema de Activación de Conductual (Behavioural Activation System, BAS en inglés) y el sistema de lucha-huída (Fight-Flight-System, FFS en inglés) (Erdle y Rushton, 2010; Pinto-Meza et al., 2009).

La activación del BIS inhibe la acción, con el fin de evitar consecuencias percibidas como potencialmente aversivas ( $\mathrm{O}^{\prime}$ Connor, Colder y Hawk, 2004), y en términos de diferencias de personalidad está más relacionado con mayores niveles de ansiedad rasgo y por consiguiente con mayores índices de neuroticismo e introversión que con niveles menores de ansiedad rasgo y estabilidad en la extraversión (Torrubia et al., 2001).

El segundo sistema de activación de comportamiento, BAS, es el sistema que responde a los comportamientos o conductas de aproximación o acercamiento ante la existencia de incentivos que así lo requieran, como las 
señales de recompensa o la no existencia de castigo. En lo concerniente a diferencias individuales de personalidad, las respuestas comandadas por el BAS están relacionadas con la dimensión de la personalidad de la impulsividad, la cual es independiente a la ansiedad y abarca, dentro del marco clásico de las personalidades de Eysenck, desde los introvertidos más estables hasta los neuróticos extravertidos (Torrubia et al., 2001).

El modelo descrito por Gray ha adquirido gran relevancia en diferentes ámbitos de estudio, entre los que podemos incluir el estudio de la personalidad y las emociones. Algunos autores como Fowles (1987), Newman (1987) o Tellegen (1985) han empleado el modelo BIS-BAS de Gray como explicación de las diferencias estables de personalidad encontradas en adultos (Torrubia et al., 2001).

Es por todo lo anterior que se considera necesario estudiar la relación de ambos constructos descritos anteriormente, motivación y sensibilidad al premio y al castigo, en un ámbito novedoso como puedan ser los estudiantes y futuros profesionales de la interpretación musical.

\section{Método}

\section{Participantes}

La muestra está compuesta por 71 estudiantes (63 hombres y 2 mujeres) del Título Superior de Música pertenecientes a 5 centros de formación superior españoles.

Todos los estudiantes son intérpretes de instrumentos de viento metal (13 trombón, 21 trompa, 25 trompeta, 8 tuba y 4 bombardino). El rango de edad abraca desde los 17 años hasta los 35 con una media de 22.81 años (D.T. $=3.39$ ).

\section{Procedimiento}

Tras una primera toma de contacto con la dirección de los centros superiores de música que solicitaron participar en el estudio, un miembro del equipo de investigación se desplazó hasta ellos para hacer un mejor acopio del cuestionario diseñado ad hoc y que debía ser respondido por los estudiantes de los centros.

\section{Instrumentos y análisis de datos}

Para el estudio de la motivación de logro se usó la escala de motivación de logro en contextos musicales establecida por Author (2014). Consiste en un cuestionario de 26 preguntas valoradas en una escala Likert de 9 puntos. El cómputo general de la misma arroja un estadístico de fiabilidad $\alpha$ de Cronbach de 0.775 . Muestra en su versión confirmatoria de 18 ítems ( $\alpha$ de Cronbach de 0.774) 5 subescalas diferenciadas: interés (4 ítems), esfuerzo (2 ítems), perfeccionismo ( 2 ítems), tarea (8 ítems) y examen (2 ítems). 
En el caso de la medición de la sensibilidad al premio y al castigo se ha empleado la versión española del cuestionario creado por Torrubia et al. en 2001 denominado The Sensitivity to Punishment and Sensitivity to Reward Questionnaire (SPSRQ). Es un instrumento con dos escalas de 24 preguntas cada una que mide ambos sistemas comportamentales, el BIS (reacciones y respuestas del sistema de inhibición de comportamientos) y el BAS (comportamientos de aproximación al premio) y que arrojó un $\alpha$ de Cronbach en la escala de castigo de 0.83 y de a 0.76 en la escala de premio. Las puntuaciones de cada escala se obtienen sumando todas las respuestas afirmativas de la misma y ambas escalas son ortogonales, independientes. En general es un instrumento fiable para medir las conductas de Inhibición de Comportamientos y las de Activación de Comportamientos por medio de sus dos escalas, la de sensibilidad al castigo y la de sensibilidad al premio. Es por lo tanto un instrumento fiable que encaja con los constructos psicológicos que se están empleando en esta investigación.

Relativo al análisis de datos, se han llevado a cabo análisis descriptivos y correlacionales con el programa informático SPSS 19.0.

\section{Resultados}

Se puede observar cómo los factores de interés, esfuerzo, tarea y exámenes presentan medias superiores a la media teórica del instrumento de evaluación de la motivación (Tabla 1). En este sentido, además estas diferencias son significativamente distintas en todos ellos. Así para el factor de interés $\mathrm{t}=22.743 ; \mathrm{p}=.000$; para esfuerzo $\mathrm{t}=12.464 ; \mathrm{p}=.000$; para exámenes $\mathrm{t}=8.591 ; \mathrm{p}=.000 ; \mathrm{y}$ para el factor tarea $\mathrm{t}=5.414 ; \mathrm{p}=.000$. Por $\mathrm{su}$ parte, para el factor de perfeccionismo presenta una media significativamente inferior a la media teórica del instrumento $(\mathrm{t}=-3.368 ; \mathrm{p}=.001)$.

Tabla1. Estadísticos descriptivos.

\begin{tabular}{|c|c|c|c|c|c|c|}
\hline & $\mathrm{N}$ & Mínimo & Máximo & Media & $\begin{array}{c}\text { Media } \\
\text { Teórica }\end{array}$ & Desv. típ. \\
\hline Interés & 71 & 18.00 & 36.00 & 31.4446 & 20 & 4.24011 \\
Esfuerzo & 71 & 5.00 & 18.00 & 14.1408 & 10 & 2.79947 \\
Perfeccionismo & 71 & 2.00 & 18.00 & 8.1549 & 10 & 4.61565 \\
Tarea & 71 & 18.00 & 64.00 & 46.0534 & 40 & 9.42193 \\
Exámenes & 71 & 6.00 & 18.00 & 12.5775 & 10 & 2.52791 \\
N válido (según lista) & 140 & & & & & \\
\hline
\end{tabular}

Siendo analizados más detenidamente los resultados, se encuentra que en torno al $21.1 \%$ de los estudiantes presentan medias alejadas de la media por la parte inferior en más de una desviación típica en el factor interés. En lo concerniente al factor esfuerzo se ve que de manera análoga al factor esfuerzo este porcentaje llega hasta el $15.5 \%$; en el factor perfeccionismo se sitúa en torno al $21.1 \%$ el porcentaje de estudiantes que suman medias inferiores a la 
media menos una desviación típica; respecto al factor tarea este porcentaje es del 9.9\%; y para el factor exámenes de al menos el $22.5 \%$.

Respecto al estudio correlacional de los distintos sub-factores de la motivación (Tabla 2), se comprueba cómo el factor interés correlaciona significativamente con los factores de esfuerzo $(r=.488 ; \mathrm{p}=.000)$ y exámenes $(\mathrm{r}=.331 ; \mathrm{p}=.005)$ mientras que lo hace de manera inversa con el factor de perfeccionismo $(r=-.270 ; p=.023)$. Asimismo, se aprecia cómo el factor tarea correlaciona a su vez de manera directamente proporcional y significativa con el factor de exámenes $(\mathrm{r}=.455 ; \mathrm{p}=.000)$.

Tabla 2. Correlaciones

\begin{tabular}{|c|c|c|c|c|c|}
\hline & Interés & Esfuerzo & Perfeccionismo & Tarea & Exámenes \\
\hline Interés & 1 & $.488^{* *}$ & $-.270^{*}$ & & $.331^{* *}$ \\
\hline Esfuerzo & $.488^{* *}$ & 1 & & & \\
\hline Perfeccionismo & $-.270^{*}$ & & 1 & & \\
\hline Tarea & & & & 1 & $.455^{* *}$ \\
\hline
\end{tabular}

Respecto al estudio de la sensibilidad al premio y al castigo se ve (Tabla 3) que la puntuación media se sitúa en el factor del premio en 10.46 puntos para el premio y en 9.73 para el castigo; siendo inferiores además estadísticamente (Premio: $\mathrm{t}=-2.875 ; \mathrm{p}=.006$; Castigo: $\mathrm{t}=-3.061 ; \mathrm{p}=.003$ ).

Tabla 3. Estadísticos descriptivos.

\begin{tabular}{|c|c|c|c|c|c|c|}
\hline & $\mathrm{N}$ & Mínimo & Máximo & Media & $\begin{array}{c}\text { Media } \\
\text { teórica }\end{array}$ & Desv. típ. \\
\hline Sensibilidad al premio & 64 & 3.00 & 20.00 & 10.4688 & 12 & 4.26119 \\
Sensibilidad al castigo & 64 & .00 & 23.00 & 9.7344 & 12 & 5.92075 \\
N válido (según lista) & 62 & & & & & \\
\hline
\end{tabular}

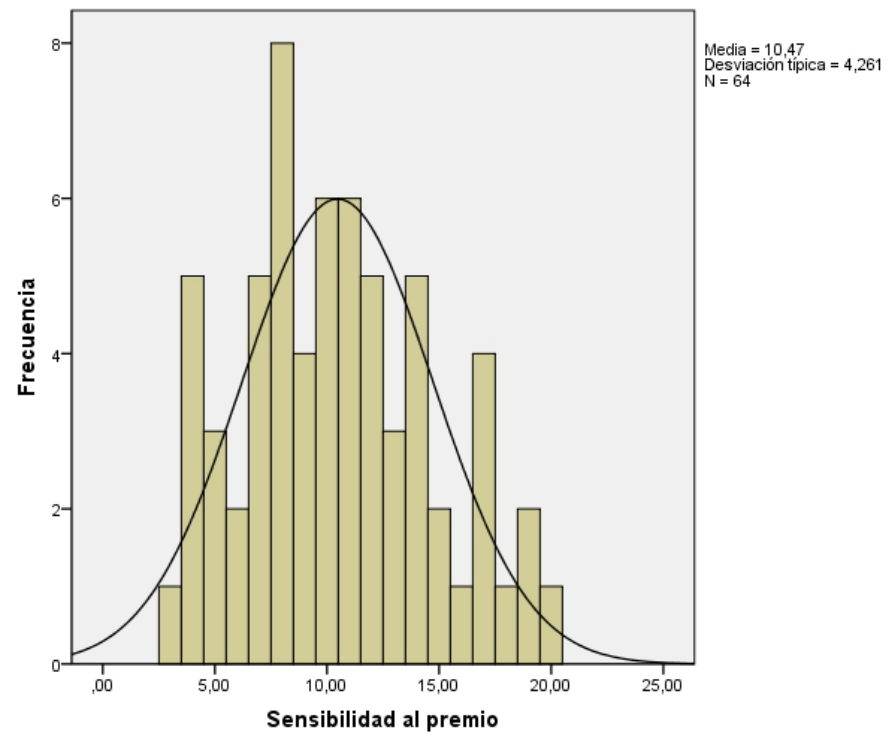

Figura 1. Distribución de la muestra. Premio. 


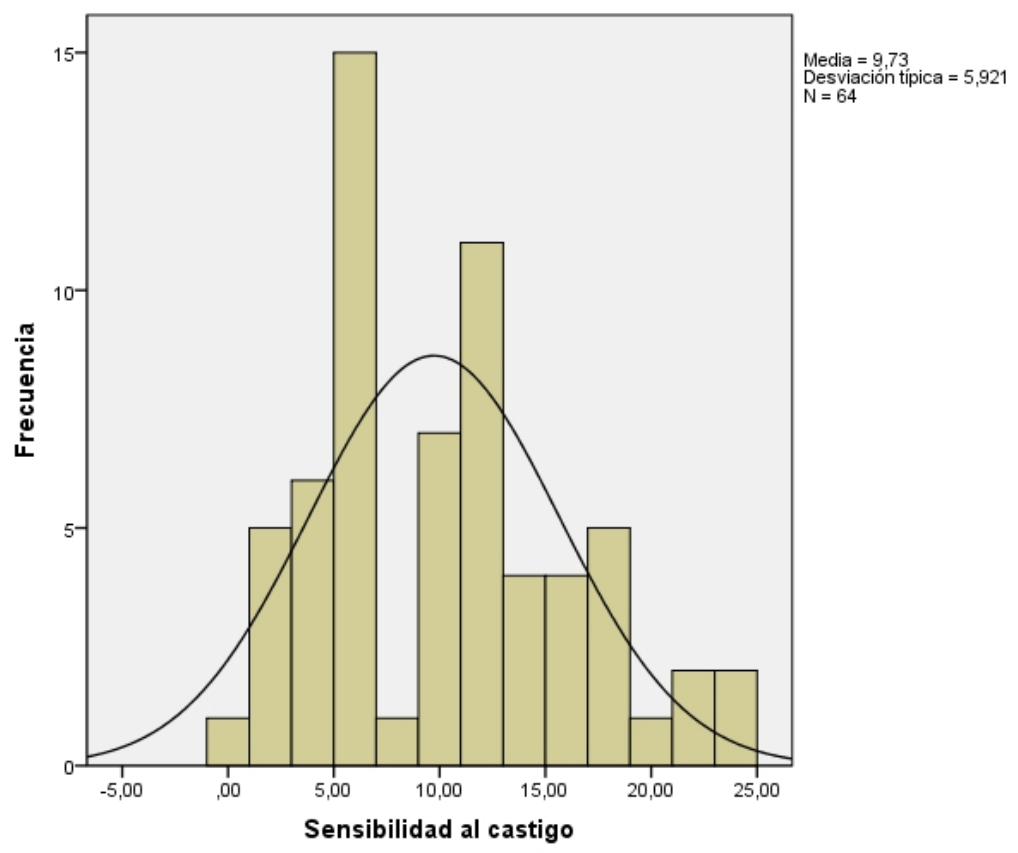

Figura 2. Distribución de la muestra. Castigo.

En este sentido se aprecia cómo en torno al 14\% de la población presenta puntuaciones medias superiores en más de una desviación típica a la media obtenida en el factor relativo al premio (Figura 1); mientras que el porcentaje es de en torno al $20 \%$ para el factor relativo a las conductas de castigo (Figura 2).

En este punto y considerando el factor motivacional como un constructo producto de la suma de los factores de interés, esfuerzo, perfeccionismo, tarea y exámenes, se considera necesario estudiar cómo la sensibilidad al premio o al castigo en su conjunto pueden explicar en un modelo de regresión con el método enter la varianza del factor general motivacional. Así, se ve que de manera significativa presenta capacidad explicativa de en torno un $11 \%$ de la varianza del factor motivacional el factor de sensibilidad al castigo $(\beta=-.373 ; \mathrm{t}=-3.001 ; \mathrm{p}=.004)$.

Tabla 4. Regresión. Método Enter. Variable dept.: Optimismo disposicional.

\begin{tabular}{|c|c|c|c|c|c|c|c|}
\hline & $\begin{array}{c}\text { Coeficientes } \\
\text { tipificados } \\
\text { Beta }\end{array}$ & $\mathrm{t}$ & $\mathrm{Sig}$. & $\mathrm{F}$ & $\mathrm{Sig}$ & $\begin{array}{c}\text { Durbin- } \\
\text { Watson }\end{array}$ & $\mathrm{R}^{2}$ \\
\hline $\begin{array}{c}\text { Sensibilidad al } \\
\text { premio }\end{array}$ & .166 & 1.334 & .187 & \multirow{2}{*}{4.710} & .012 & 2.207 & .108 \\
\hline $\begin{array}{c}\text { Sensibilidad al } \\
\text { castigo }\end{array}$ & -.373 & -3.001 & .004 & & & \\
\hline
\end{tabular}




\section{Conclusión}

Se ha podido comprobar cómo el constructo de la motivación de logro es un constructo multifactorial (interés, esfuerzo, perfeccionismo, tarea y exámenes) en el cual cada uno de sus factores presenta un papel definido y separado del resto. En este sentido, esta aproximación se enlaza con investigaciones previas como la de Author, Casanova y Orejudo (2016), en las cuales también se estudia la motivación en función, en este caso, de variables más relacionadas con la práctica pedagógica. Así, aquí ha quedado comprobado cómo los componentes de la sensibilidad al premio y al castigo, tan relacionados con la ansiedad como describen Torrubia et al. (2001), presentan un potencial explicativo digno de tener en cuenta en lo que a la motivación se refiere. Del mismo modo, no queda claro, como también ocurre en Author, Orejudo, Casanova y Aparicio (2018) el papel que pueda desempeñar en el constructo motivacional en conjunto la sensibilidad al premio. Este hecho debe ser considerado como susceptible de más estudios que provean de evidencias científicas suficientes para poder aclarar esta circunstancia. Así, la consideración de la motivación, especialmente la relacionada con las tareas específicas, como, hasta cierto punto, producto de la sensibilidad al castigo que tengan los estudiantes, debe ser un hecho que no deje indiferente a la comunidad pedagógica en general; todo ello de cara al mejor diseño de programas educativos y de su concreción en el aula.

\section{References:}

1. Carver, C. S. (2006). Approach, avoidance, and self-regulation of affect and action. Motivation and Emotion, 30, 105-110.

2. Cloninger, C. R. (1987). A systematic method of clinical description and classification of personality variants: A proposal. Archives of General Psychiatry, 44, 573-588.

3. Davidson, R. J. (1984). Affect, cognition, and hemispheric specialization. In C. E. Izard, J. Kagan \& R. Zajonc (Eds.), Emotion, cognition, and behavior (pp. 320-365). New York, NY: Cambridge University Press.

4. Davidson, R. J. (1988). EEG measures of cerebral asymmetry: Conceptual and methodological issues. International Journal of Neuroscience, 39, 71-89.

5. Davidson, R. J. (1995). Cerebral asymmetry, emotion, and affective style. In R. J. Davidson \& K. Hugdahl (Eds.), Brain asymmetry (pp. 361-387). Cambridge, MA: MIT Press.

6. Davidson, R. J. (1998). Affective style and affective disorders: Perspectives from affective neuroscience. Cognition and Emotion, 12, 307-330. 
7. Depue, R. A. \& Collins, P. F. (1999). Neurobiology of the structure of personality: Dopamine, facilitation of incentive motivation, and extraversion. Behavioral and Brain Sciences, 22(3), 491-517.

8. Depue, R. A., Krauss, S., \& Spoont, M. (1987). A two-dimensional threshold model of seasonal bipolar affective disorder. In D. Magnusson \& A. Ohman (Eds.), Psychopathology: An interactionist perspective. New York, NY: Academic Press.

9. Elliot, A. J. \& Church, M. A. (1997). A hierarchical model of approach and avoidance achievement motivation. Journal of Personality and Social Psychology, 72, 218-222.

10. Erdle, S. \& Rushton, J. P. (2010). The General Factor of Personality, BISBAS, expectancies of reward and punishment, self-esteem, and positive and negative affect. Personality and Individual Differences, 48(6), $762-$ 766. doi:10.1016/j.paid.2010.01.025

11. Ericsson, K. A., Krampe, R. T., \& Tesch-Römer, C. (1993). The role of deliberate practice in the acquisition of expert performance. Psychological Review, 100, 363-406.

12. Eysenck H. J. (1985). Incubation theory of fear/anxiety. In S. Reiss \& R. R. Bootzin (Eds.), Theoretical foundations in behavior therapy (pp. 83106). New York, NY: Plenum Press.

13. Fowles, D. C. (1980). The three arousal model: Implications of Gray's two-factor learning theory for heart rate, electrodermal activity, and psychopathy. Psychophysiology, 17, 87-104.

14. Fowles, D. C. (1987). Application of a behavioural theory of affect to the concepts of anxiety and impulsivity. Journal of Research in Personality, 21, 417-435.

15. Gray, J. A. (1981). A critique of Eysenck's theory of personality. In H. J. Eysenck (Ed.), A model for personality (pp. 246-276). Berlin: SpringerVerlag.

16. Gray, J. A. (1986). Discussions arising from Cloninger, C. R.: A unified biosocial theory of personality and its role in the development of anxiety states. Psychiatric Developments, 3, 167-226.

17. Gray, J. A. (1987). Perspectives on anxiety and impulsivity: A commentary. Journal of Research in Personality, 21, 493-509.

18. Gray, J. A. (1990). Brain systemsthat mediate both emotion and cognition. Cognition and Emotion, 4, 269-288.

19. Gray, J. A. (1994a). Personality dimensions and emotion systems. In P. Ekman \& R. J. Davidson (Eds.), The nature of emotion: Fundamental questions (pp. 329-331). New York, NY: Oxford University Press.

20. Gray, J. A. (1994b). Three fundamental emotion systems. In P. Ekman \& R. J. Davidson (Eds.), The nature of emotion: Fundamental questions (pp. 243-247). New York, NY: Oxford University Press. 
21. Hernangómez, L. \& Fernández, C. (2012). Psicología de la personalidad $y$ diferencial. Madrid, Spain: CEDE.

22. Manassero, M. A. \& Vázquez, A. (1998). Validación de una escala de motivación de logro. Psicothema, 10(2), 333-351.

23. Newman, J. P. (1987). Reaction to punishment in extroverts and psichopaths: Implications for the impulsive behaviour of disinhibited individuals. Journal of Research in Personality, 21, 464-480.

24. O’Connor, R. M., Colder, C. R., \& Hawk, L. W. (2004). Confirmatory factor analysis of the Sensitivity to Punishment and Sensitivity to Reward Questionnaire. Personality and Individual Differences, 37(5), 985-1002. doi:10.1016/j.paid.2003.11.008

25. Pinto-Meza. A., Suárez, D., Caseras, X., Haro, J. M., Serrano-Blanco, A., \& Torrubia, R. (2009). Evaluación telefónica de los sistemas de activación e inhibición conductual. Revista de Psiquiatría y Salud Mental, 2(2), 6671.

26. Shehan-Campbell, P. (1995). Of Garage bands and song-getting: The musical development of young rock musicians. Research Studies in Music Education, 4, 12-20.

27. Tellegen, A. (1985). Structure of mood and personality and their relevance to assessing anxiety, with an emphasis of self-report. In A. H. Tuma \& J. Mason (Eds.), Anxiety and anxiety disorders (pp. 681-706). Hillsdale, NJ: Lawrence Erlbaum Associates.

28. Torrubia, R., Ávila, C., Moltó, J., \& Caseras, X. (2001). The sensitivity to punishment and sensitivity to reward questionnaire (SPSRQ) as a measure of Gray's anxiety and impulsivity dimensions. Personality and Individual Differences, 31(6), 837-862. doi:10.1016/S0191-8869(00)00183-5

29. Walser, R. (1993). Running with the Devil: Power, Gender, and Madness in Heavy Metal Music. Middletown, Connecticut (USA): Wesleyan University Press.

30. Weiner, B. (1986a). An Attributional Theory of Motivation and Emotion. New York, NY: Springer-Verlag.

31. Weiner, B. (1986b). Attribution, Emotion, and Action. In R. M. Sorrentino \& E. T. Higgins (Eds.), Handbook of Motivation and Cognition, Volume 1: Foundations of Social Behavior (pp. 281-312). New York, NY: Guidford Press.

32. Weiner, B. (2000). Interpersonal and intrapersonal theories of motivation from an attributional perspective. Educational Psychology Review, 12, 114.

33. Author, F. J. (2014). Variables psicológicas y pedagógicas como predictoras de la ansiedad escénica en estudiantes de Grado Superior de Música de España. Tesis doctoral. Zaragoza: Universidad de Zaragoza. 
34. Author, F. J., Casanova, O., \& Orejudo, S. (2016). Ansiedad escénica y constructos psicológicos relacionados. Estudiantes de cinco conservatorios superiores de música españoles. RIEM. Revista Internacional de Educación Musical, 4, 13-24. doi:10.12967/RIEM2016-4-p013-024

35. Author, F. J., Orejudo, S., Casanova, O., \& Aparicio, L. (2018). Music Performance Ansiety in adolescence and early adulthood: Its relation with the age of onset in musical training. Psychology of Music, 46(1), 18-32. doi: $10.1177 / 0305735617691592$ 